This item was submitted to Loughborough's Research Repository by the author.

Items in Figshare are protected by copyright, with all rights reserved, unless otherwise indicated.

\title{
A game theoretic optimization framework for home demand management incorporating local energy resources
}

PLEASE CITE THE PUBLISHED VERSION

https://doi.org/10.1109/TII.2015.2390035

PUBLISHER

(C) IEEE

VERSION

AM (Accepted Manuscript)

LICENCE

CC BY-NC-ND 4.0

\section{REPOSITORY RECORD}

Zhu, Ziming, Sangarapillai Lambotharan, Woon Hau Chin, and Zhong Fan. 2015. "A Game Theoretic Optimization Framework for Home Demand Management Incorporating Local Energy Resources". Loughborough University. https://hdl.handle.net/2134/34587. 


\section{A Game Theoretic Optimization Framework for Home Demand Management Incorporating Local Energy Resources}

\begin{abstract}
Facilitated by advanced ICT infrastructure and optimization techniques, smart grid has the potential to bring significant benefits to the energy consumption management. This paper presents a game theoretic consumption scheduling framework based on the use of mixed integer programming to schedule consumption plan for residential consumers. In particular, the optimization framework incorporates integration of locally generated renewable energy in order to minimise dependency on conventional energy and the consumption cost. The game theoretic model is designed to coordinatively manage the scheduling of appliances of consumers. The Nash equilibrium of the game exists and the scheduling optimization converges to an equilibrium where all consumers can benefit from participating in. Simulation results are presented to demonstrate the proposed approach and the benefits of home demand management.
\end{abstract}

Index Terms-Smart grids, home demand management, consumption scheduling optimization, game theory.

\section{INTRODUCTION}

Demand management is the key to operational efficiency and reliability of electric grids [1]. The future power grid is expected to bring significant improvements to energy generation and dispatch. The direction of power flow will no longer be just downhill from the bulk power plants to consumers. Power flow can originate from any energy generation sources and could end up anywhere on the grid. As micro level local renewable energy generation such as rooftop solar cells and the use of hybrid electric vehicles become popular, electricity can be generated and stored by consumers and can be released to the grid when necessary [2]. In the future, consumers will have the flexibility to consume energy from various sources and make the best use of locally generated energy. Demand management for smart grids needs to be efficient in terms of optimizing energy demand and supply [3]. Recent advances in information and communication technologies (ICT) have enabled real time monitoring and control of the grid's operational conditions. In particular, the utility operators and consumers can communicate and coordinate bi-directional load control and consumption optimizations [4].

Consumption scheduling is one of the important fundamental approaches for demand management. It is achieved by changing the normal electricity consumption patterns of end consumers over time and reducing the energy demand at peak times when whole sale market price is high or when system reliability is jeopardized [5]. Techniques such as peak clipping and flexible load shaping and shifting have been in place for many decades [6]. However, centralised control and scheduling of large scale of appliances, for example, ON/OFF switching or change of operational parameters by the grid operators appear impractical due to unacceptable level of delays and data traffic for exchanging control messages. Instead, utility operators can perform indirect demand management according to various demand and supply conditions by encouraging proactive participation of consumers. Indirect demand management operates through incentives, such as pricing, energy trading/brokering and even social interaction [7], [8]. Attractive price plans containing changeable rates, for example block based time-of-use (ToU) pricing, critical peak pricing (CPP) [9] and dynamic/real-time pricing schemes [10], [11], provide consumers economic incentives to manage their energy consumption efficiently and to reap financial benefits. Various candidate electricity market models have been studied in [12]-[14]. Considering that households are directly responsible for actual energy consumption and management of local energy generation, consumer oriented proactive and distributed consumption scheduling is very attractive. A number of households in a neighbourhood could participate locally to reduce the peak load based on very minimum level of instruction from utility operators. Hence the indirect demand management can benefit from low delay and low data traffic for controlling the appliances.

In order to facilitate consumption scheduling, it is essential to develop appropriate mathematical optimization algorithms for energy consumption. There are emerging works on optimal energy consumption based on both centralised and distributed optimization algorithms. For example, a centralised phase management scheme has been developed in [15] that provided optimal time shifts for periodic loads in order to provide peak power reduction over a limited time horizon. A heuristic based evolutionary algorithm (EA) was proposed in [16] for solving day-ahead load shifting problems. The work in [17] proposed an integrated solution to predict and schedule electricity demand in the locality based on user preferences and social/environmental factors. The work in [18] developed a stochastic optimization method for residential, commercial and industrial energy consumers and distributed energy generators in a microgrid scenario. Apart from optimizations with realvalued variables, integer and mixed integer programming (MIP) techniques have also been used for demand management in smart grids. The work in [19] studied the implementation of MIP in scheduling optimization of a mircogrid system in order 
to reduce both the operational cost and carbon emission. The authors of [20] further proposed a stochastic MIP framework incorporating fuzzy decision making in order to obtain socially optimal solutions.

Game theory is a powerful mechanism for understanding and modelling mathematically the interaction of various rational decision makers (known as players). Game theoretic methods have been widely applied in resource competing and social welfare optimization scenarios [21]. It is very suitable for analysing the interaction of consumers and utility operators in energy demand management, when considering the distributed nature of the operation of the smart grids and the need for low complexity algorithms [22]. Mathematical optimization techniques and game theoretic framework have the potential to provide very efficient consumer incentive based distributed consumption scheduling [23]. For example, an energy scheduling game based on convex optimization technique was proposed in [24] to schedule power consumption of various appliances. Constraints such as minimum standby power and maximum operating power of the appliances were formulated using a convex optimization framework. The framework has been extended to multiple households scenario and a consumption scheduling game was proposed to enable consumers to respond to energy price information. In [25], a two-layer game theoretic framework was established. At the lower level, appliances are scheduled for energy consumption for each household. At the upper level, a dynamic differential game was used to capture the scheduling interaction among different households. The authors of [26] proposed a leaderfollower Stackelberg game with utility companies as leaders and end consumers as followers to maximise the revenue of each utility company and the payoff of consumers. A distributed convergence algorithm was developed that requires only local information.

Many appliances have their own fixed energy consumption patterns. It means once such an appliance is scheduled for operation, changing the power consumption during the operational period may not be possible or unattractive. Considering this requirement, a game theoretic consumption scheduling optimization framework based on mixed integer programming is proposed in this paper. The proposed framework incorporates integration of local energy resources in order to reduce dependency on conventional energy and the cost of energy consumption. One of the advantages of the proposed approach is that the computational complexity can be distributed among the individual home demand management units by decomposing the large scale centralised optimization using a decentralised game theoretic method. In particular, by formulating the scheduling optimization as a potential game, it is possible to achieve a Pareto optimal Nash equilibrium where all consumers can benefit from participating in. In this paper, we will focus on consumption scheduling of residential consumers. However, the proposed framework can be extended to electric vehicle (EV) charging applications, as well as distributed generation and dispatch systems.

The rest of the paper is organised as follows. The detailed formulation of the MIP scheduling framework is proposed in Section II. In Section III, the consumption scheduling game

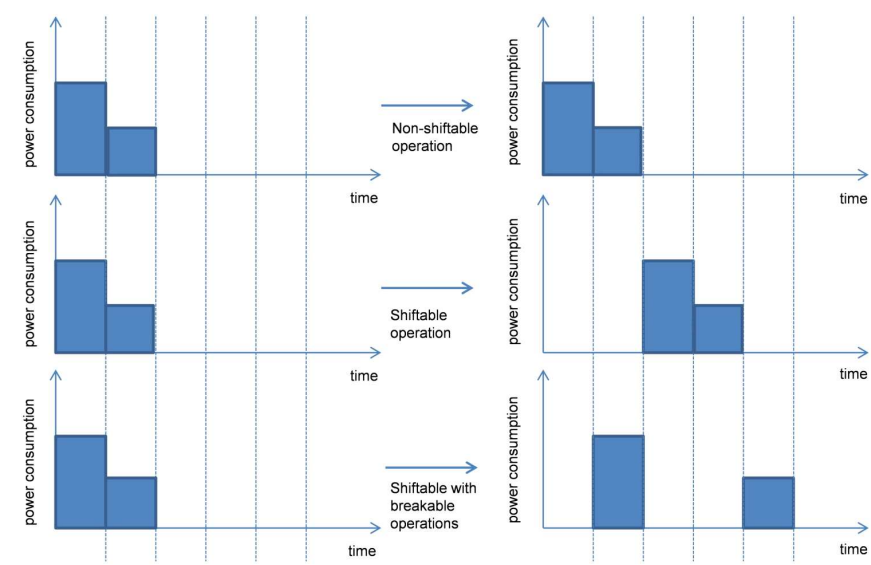

Fig. 1: Illustrative examples of different types of operation of appliances.

among individual consumers based on the optimization framework is formulated. The existence of Nash equilibrium, which promises the game a stable solution, and the participating behaviour of consumers are analysed. Numerical simulation results are presented in Section IV and conclusions are drawn in Section V.

\section{MIP BASED HOME CONSUMPTION SCHEDULING OPTIMIZATION}

A mathematical optimization technique is described for scheduling daily energy consumption of appliances at household level with the aim of reducing peak accumulated consumption and the cost of energy for consumers. In particular, the households are able to draw power from both the main grid under a given price plan as well as from locally generated renewable energy sources. The optimization is expected to maximise the usage of locally generated energy while drawing energy from the main grids optimally whenever required.

\section{A. Classification of appliances}

The household appliances are classified into two groups namely non-shiftable and shiftable appliances [27].

Non-shiftable appliances are those appliances for which scheduling is not possible. For example, a fridge is expected to operate continuously throughout the day, and a central heating system needs to be in operation whenever it is required by the consumer. The operations of these appliances are strictly dominated by user comfort and convenience. Shifting operations of these appliances can bring considerable discomfort to consumers, hence not allowed. The reader is referred to [28] for alternative approaches for managing user preferences and benefits. The second class of appliances is defined as shiftable appliances whose operations can be scheduled during certain predefined periods. Appliances such as washing machines and storage heating systems belong to this class. The consumers can tolerate the shift (postponing) of the operations of these appliances as long as the required operations will be finished within a preferred time period and will yield financial benefits.

It is necessary to distinguish elastic and inelastic demands within the class of shiftable appliances. Elastic demand refer to appliances whose operation can be performed noncontinuously and the whole operation time can be divided 
into several non-continuous tasks. Preemptive consumption scheduling is possible. For example, the operation of a water tank boiler can be broken into several heating tasks throughout the day and certain energy consumption is required for each task. Mathematically, the scheduling mechanism will treat every task as an individual appliance that can be scheduled to operate at different times. Inelastic demand means the energy consumption pattern of the appliances cannot be changed during operation. This means once the appliance starts its operation, it will need to consume energy according to its own consumption pattern until the operation is finished. A washing machine can be considered within this class and the scheduling for these appliances are non-preemptive [29]. For appliances which have pre-programmed non-preemptive operations, the total operation should be scheduled as a whole with the energy supply according to the appliance's consumption pattern. Fig. 1 illustrates typical operations and energy consumption of various classes of appliances.

\section{B. The optimization objective}

Let us consider a daily pricing scenario where the cost of energy is determined as a function of time and energy consumption generated by all subscribing users in the billing area. The total cost of the energy consumption of all users is represented by a vector $\mathbf{C}=\left[C_{1}, C_{2}, \ldots, C_{t}, \ldots, C_{T}\right]^{T}$, where $t$ is the time-of-use parameter which is also the time slot indicator for the scheduling optimization. $T$ accounts for the time resolution, for example, $T=24$ and $T=1440$ represent respectively the hourly and minute based schedulings. The cost of energy $C_{t}$ at time $t$, is written as a function of the accumulated consumption of all consumers $L_{t}$ as follows,

$$
C_{t}=\omega_{t} L_{t}^{2}+\omega_{t} L_{t}+\phi_{t},
$$

where $\omega_{t}$ is the basic TOU unit rate which can take various values for different time slots and $\phi_{t}$ is an independent standard charge at time $t$, e.g., additional fees for critical peak events [30]. Denote the scheduled daily consumption result for individual consumer $n \in \mathbb{N}$ as $\mathbf{L}_{n}=\left[L_{n, 1}, L_{n, 2}, \ldots, L_{n, T}\right]^{T} \in$ $\mathbb{R}^{T \times 1}$, where $L_{n, t}$ is the energy consumption of consumer $n$ at time $t$, and $\mathbb{N}$ denotes the set of $N$ consumers. Hence $L_{t}=\sum_{n \in \mathbb{N}} L_{n, t}$. Denote $\mathbf{L}=\sum_{n \in \mathbb{N}} \mathbf{L}_{n} \in \mathbb{R}^{T \times 1}$ as the consumption scheduling profile of the area considered in the optimization. The cost of energy consumption of the day can be calculated as

$$
C(\mathbf{L})=\sum_{t} C_{t}=\sum_{t}\left(\omega_{t}\left(\sum_{n \in \mathbb{N}} L_{n, t}\right)^{2}+\omega_{t}\left(\sum_{n \in \mathbb{N}} L_{n, t}\right)+\phi_{t}\right) .
$$

The pricing plan can be viewed as a continuous function approximating the existing stepwise (multi-step) pricing models adopted in the current electricity markets [31]-[33] and [24]. However, most of these models were designed to charge individual consumers at different rates according to their monthly/yearly accumulated energy consumption in a bid to encourage them to save energy. These models have limited leverage on customers to reduce the accumulated consumption of the area at various times. By issuing ToU pricing, utility operators can enforce high rates during high demand periods. The consumers will be motivated to shift their consumption as much as possible from the peak ToU periods. In particular, it can be observed in (2) that the cost is not only proportional to the ToU rate $\omega_{t}$ but also increases quadratically with the accumulated consumption $L_{t}$. The price per unit of consumption (assumed $\phi_{t}=0$ ) at time $t$ has the value of $C_{t} / L_{t}=\omega_{t} L_{t}+\omega_{t}$. For consumer $n$, the cost of energy at time $t$ is

$$
C_{n, t}=\left(\omega_{t} L_{t}+\omega_{t}\right) L_{n, t} .
$$

This relationship implies that individual's cost of energy consumption depends on the demand of all consumers. Hence, consumers have incentive to reduce the accumulated consumption at every time slot over the day as this will in turn reduce their energy costs. As all consumers are assumed to be rational, coordination among consumers is automatically achieved in order to avoid overlapping consumption at various times. These activities are modeled as a constrained game in Section III.

For a given accumulated consumption profile $\tilde{L}_{n, t}=$ $\sum_{i \in \mathbb{N}, i \neq n} L_{i, t}$ of all consumers other than $n$, the consumer $n$ aims to optimise the following,

$$
C_{(n)}(\mathbf{L})=\sum_{t}\left(\omega_{t}\left(L_{n, t}+\tilde{L}_{n, t}\right)^{2}+\omega_{t}\left(L_{n, t}+\tilde{L}_{n, t}\right)+\phi_{t}\right) .
$$

It is highlighted again that by reducing the total cost $C$ in (2), each consumer aims to reduce the cost of his/her energy use as in (3). Subscript $(n)$ is used to explicitly indicate consumer $n$ 's contribution of the optimization of cost.

In the proposed mechanism, it is assumed that the energy consumption from local energy sources results into zero cost to the consumer's electricity bill. Therefore the consumers will attempt to make full use of local energy supply to minimise dependency on conventional energy and to optimise the consumption cost charged by the utility operator. Consumers will reserve any unused/surplus local energy for future use or may be able to release it to the main grid and generate revenue [34]. However this is not considered in the proposed optimization framework.

\section{Constraints Formulation}

The consumption requirements of various appliances and the use of local energy are formulated as various constraints in the optimization problem. Consider consumer $n$ has a set of home appliances $\mathbb{A}_{n}$. An appliance $a \in \mathbb{A}_{n}$ has a total daily energy consumption requirement of $l_{n, a}$. The vector $\mathbf{x}_{n, a}=$ $\left[x_{n, a, 1}, x_{n, a, 2}, \ldots, x_{n, a, T}\right]^{T}$ is used to denote the scheduled energy consumption over the day for the appliance $a$. The parameter $x_{n, a, t}$ denotes the intended energy consumption of the appliance $a$ of user $n$ at time $t$, and hence $\mathbf{L}_{n}=\sum_{a} \mathbf{x}_{n, a}$. Suppose the appliance $a$ is required to operate between time slots $t_{n, a, s}$ and $t_{n, a, f}$, its total energy requirement can be ensured by the constraint

$$
\sum_{t=t_{n, a, s}}^{t_{n, a, f}} x_{n, a, t}=l_{n, a}, \forall a \in \mathbb{A}_{n} .
$$

A shiftable appliance $a \in \mathbb{A}_{n, s} \subset \mathbb{A}_{n}$ can 
have a predefined energy consumption pattern $\mathbf{p}_{n, a}=$ $\left[p_{n, a, 1}, p_{n, a, 2}, \ldots, p_{n, a, T}\right]^{T}$. In this case only the optimal starting time can be scheduled. The scheduling result $\mathbf{x}_{n, a}$ can be viewed as one of the cyclic shifts of the pattern $\mathbf{p}_{n, a}$ [35]. All possible shifts for the vector $\mathbf{p}_{n, a}$ can be put together in a matrix form as

$$
\mathbf{P}_{n, a}=\left[\begin{array}{ccccc}
p_{n, a, 1} & p_{n, a, T} & \ldots & p_{n, a, 3} & p_{n, a, 2} \\
p_{n, a, 2} & p_{n, a, 1} & \ldots & p_{n, a, 4} & p_{n, a, 3} \\
\vdots & \vdots & \ddots & \vdots & \vdots \\
p_{n, a, T} & p_{n, a, T-1} \ldots & p_{n, a, 2} & p_{n, a, 1}
\end{array}\right], \forall a \in \mathbb{A}_{n, s} .
$$

A binary integer vector $\mathbf{s}_{n, a}=$ $\left[s_{n, a, 1}, s_{n, a, 2}, \ldots, s_{n, a, T}\right]^{T}, s_{n, a, t} \in\{0,1\}$ is defined as the switch control of the energy consumption from the main supply for the shiftable appliance $a \in \mathbb{A}_{n, s}$. There is only one non-zero element in the vector $\mathbf{s}_{n, a}$ which is equal to one. Hence the vector $\mathbf{s}_{n, a}$ is an optimization parameter which chooses appropriate column from $\mathbf{P}_{n, a}$ to optimise the energy consumption, i.e.,

$$
\mathbf{x}_{n, a}=\mathbf{P}_{n, a} \mathbf{s}_{n, a}, \quad \sum_{t} s_{n, a, t}=1, \forall a \in \mathbb{A}_{n, s} .
$$

For a non-shiftable appliance with a strictly inflexible operation requirement, the consumption scheduling should be fixed as required by the consumer. Suppose there is a non-shiftable appliance $a \in \mathbb{A}_{n, f}, \mathbb{A}_{n, f} \subset \mathbb{A}_{n}$, with the energy consumption pattern $\mathbf{p}_{n, a}$. Since both the value and the position of the elements in $\mathbf{p}_{n, a}$ cannot be changed, the scheduling constraint can be written as

$$
\mathbf{x}_{n, a}=\mathbf{p}_{n, a}, \forall a \in \mathbb{A}_{n, f} .
$$

The scheduler is able to draw energy optimally from either the main grid or local energy sources for every appliances. Considering that local energy resources, such as wind and solar, could be intermittent, local energy should be scheduled only when its available capacity is sufficient for supplying power during the appliance's operation period. A separate vector $\mathbf{s}_{n, a}^{l}$ is introduced to indicate the switching parameter for the local energy consumption for appliance $a$. Now the appliance has two switching parameters to determine its operation time and the source of energy. The scheduling constraint can be formulated as follows,

$$
\begin{aligned}
& \mathbf{s}_{n, a}+\mathbf{s}_{n, a}^{l} \leq \mathbf{1}, \\
& \mathbf{x}_{n, a}=\mathbf{P}_{n, a} \mathbf{s}_{n, a}+\mathbf{P}_{n, a} \mathbf{s}_{n, a}^{l}, \forall a \in \mathbb{A}_{n, s},
\end{aligned}
$$

where $\mathbf{1}=[1, \ldots, 1]^{T}$.

As discussed before, the proposed mechanism treats every part of a breakable operation as an individual appliance operating in different time slots. Suppose the operation of an appliance can be decomposed into $\mathbb{K}_{a}$ scheduling tasks (appliances), $\mathbb{K}_{a}=\left\{a_{1}, \ldots, a_{k}\right\}$. The total consumption scheduling can be denoted as the sum of all individual tasks, i.e., $\mathbf{x}_{n, a}=$ $\mathbf{x}_{n, a_{1}}+\mathbf{x}_{n, a_{2}}+\cdots+\mathbf{x}_{n, a_{k}}$. Each task has its consumption requirement of $l_{n, a_{k}}$ and a consumption profile $\mathbf{p}_{n, a_{k}}$ which can be decomposed from those of the original appliance. Two switches are used to schedule each consumption task $\mathbf{x}_{n, a_{k}}$ and formulate them into the following set of constraints as similar to (9),

$$
\begin{aligned}
& \mathbf{s}_{n, a_{k}}+\mathbf{s}_{n, a_{k}}^{l} \leq \mathbf{1}, \\
& \mathbf{x}_{n, a_{k}}=\mathbf{P}_{n, a_{k}} \mathbf{s}_{n, a_{k}}+\mathbf{P}_{n, a_{k}} \mathbf{s}_{n, a_{k}}^{l}, \\
& \sum_{k \in \mathbb{K}_{a}} \mathbf{s}_{n, a_{k}} \leq \mathbf{1}, \sum_{k \in \mathbb{K}_{a}} \mathbf{s}_{n, a_{k}}^{l} \leq \mathbf{1}, \forall k \in \mathbb{K}_{a}, \forall a \in \mathbb{A}_{n, s} .
\end{aligned}
$$

The constraint (12) ensures that the divided tasks are operating in different time slots. Actually, an appliance with nonbreakable operation can be viewed as a particular appliance with only one scheduleable task.

Finally, the scheduled energy consumption from local energy supply for all appliances must not exceed the consumer's local generation and storage capacity $y_{n}$, i.e.,

$$
\sum_{a \in \mathbb{A}_{n}} \sum_{k \in \mathbb{K}_{a}} \mathbf{P}_{n, a_{k}} \mathbf{s}_{n, a_{k}}^{l} \leq y_{n}
$$

\section{The Local scheduling optimization problem}

Based on the above formulations, the optimization problem for the individual consumer $n$ as minimization of utility cost as defined in (4) through optimum scheduling of energy consumption, subject to the consumption requirements of all appliances and the capacity of local energy resource, is formulated as follows:

$$
\begin{aligned}
& \min _{t \in \mathbb{R}^{+}} C_{(n)}(\mathbf{L}) \\
& x_{n, a_{k}, t \in \mathbb{R}^{+}}, \mathbf{s}^{l}, \mathbb{Z}^{+T \times} \\
& \text { s.t. } \sum_{a \in \mathbb{A}_{n}} \sum_{k \in \mathbb{K}_{a}} x_{n, a_{k}, t}=L_{n, t}, \\
& \sum_{k \in \mathbb{K}_{a}} \sum_{t=t_{n, a_{k}, s}}^{t_{n, a_{k}, f}} x_{n, a_{k}, t}=l_{n, a}, \forall a \in \mathbb{A}_{n}, \\
& \mathbf{x}_{n, a}=\mathbf{p}_{n, a}, \forall a \in \mathbb{A}_{n, f}, \\
& \mathbf{x}_{n, a_{k}}=\mathbf{P}_{n, a_{k}} \mathbf{s}_{n, a_{k}}+\mathbf{P}_{n, a_{k}} \mathbf{s}_{n, a_{k}}^{l} \text {, } \\
& \mathbf{s}_{n, a_{k}}+\mathbf{s}_{n, a_{k}}^{l} \leq \mathbf{1}, \sum_{t} s_{n, a_{k}, t}=1, \sum_{t} s_{n, a_{k}, t}^{l}=1 \text {, } \\
& \sum_{k \in \mathbb{K}_{a}} \mathbf{s}_{n, a_{k}} \leq \mathbf{1}, \sum_{k \in \mathbb{K}_{a}} \mathbf{s}_{n, a_{k}}^{l} \leq \mathbf{1}, \\
& \sum_{a \in \mathbb{A}_{n}} \sum_{k \in \mathbb{K}_{a}} \mathbf{P}_{n, a_{k}} \mathbf{s}_{n, a_{k}}^{l} \leq y_{n}, \forall k \in \mathbb{K}_{a}, \forall a \in \mathbb{A}_{n, s} .
\end{aligned}
$$

The optimization in (14) is a mixed integer programming which can be solved using branch and bound method [36]. The method obtains the global solution by dividing the original MIP problem into a number of smaller subproblems. It uses linear programming relaxation and determines how good a solution at each subproblem by splitting the subproblem further, until the optimal solution is reached. Branch and bound algorithms have been applied in a wide variety of integer optimization problems and are widely accepted as efficient for solving small-medium size problems with tens of variables. However, in the worst case the complexity of branch and bound method can increase exponentially with 
the problem size. In addition, in order to obtain optimum scheduling using (14), $\tilde{L}_{n, t}$ is required, i.e., the optimization requires coordination among consumers. In this paper, the scheduling of consumers are analysed as a strategic game.

\section{GAME THEORETIC SCHEDULING APPROACH}

\section{A. Game components}

In this section, a game theoretic model for the consumption scheduling of various consumers under a pricing plan as described in (2) is presented. In game theory, a non-cooperative game is defined as a strategic interaction of rational players consisting of three main components, namely players, strategies and payoffs [37]. The constrained consumption scheduling optimization game components are as follows:

Players: The set of $N$ distributed energy consumers $\mathbb{N}$ in the same billing area.

Strategies: The daily consumption scheduling plan that each player $n$ chooses to play the game, i.e., $\mathbf{L}_{n} \in \mathbb{S}_{n}$ is used to represent the strategy chosen by player $n$, where $\mathbb{S}_{n}$ denotes the strategy space of player $n$. The strategy space of all players is defined as $\mathbb{S}=\prod_{n \in \mathbb{N}} \mathbb{S}_{n}$.

Payoffs: The payoff of each player is the negative of the cost of energy consumption charged by the utility company. The function $\mathfrak{u}_{n}\left(\mathbf{L}_{n}, \mathbf{L}_{-n}\right), \mathfrak{u}_{n}: \mathbb{S} \mapsto \mathbb{R}$ is used to represent the payoff for a chosen strategy of player $n$, given the strategy choices $\mathbf{L}_{-n} \in \mathbb{S}_{-n}$ of all other players (i.e., the energy consumption profiles of all other consumers), where $\mathbb{S}_{-n}=\prod_{i \in \mathbb{N}, i \neq n} \mathbb{S}_{i}$.

Every consumer will want to minimise only his/her energy cost and will be tempted to select a consumption schedule that maximises his/her payoff $\mathfrak{u}_{n}$ as the best strategy in response to the price plan and other players' chosen strategies. it can be claimed that consumers may not be able to achieve the lowest possible cost (i.e., maximal payoff) unless they participate in the game. Suppose all players schedule operation of appliances in isolation to respond to the price, then it will lead all players scheduling their consumption of energy when the ToU rate is low. This will increase the chances of all consumers operate the appliances at the same time and will result into high accumulated consumption. This will in turn increase the cost of energy to every consumer. Hence, consumers will attempt to schedule their consumption to minimise the total group cost $C$ using the optimization framework in (14). Individual's optimal scheduling is achieved when the cost of all consumers has reached its minimal. However, each consumer will need to pay only their share of the cost. This is an analogy to network utility maximization situation where individuals benefit the most when the whole network is optimised [38]. In this case, the payoff function for all players can be represented by (4), i.e., $\mathfrak{u}_{n}\left(\mathbf{L}_{n}, \mathbf{L}_{-n}\right)=-C_{(n)}, \forall n \in \mathbb{N}$. The game is expected to provide a more balanced scheduling result and reduced cost, as demonstrated in the simulation section.

Note that in the scheduling game defined above, players must solve constrained optimizations to obtain their optimal payoffs and strategies. Therefore, it is necessary to define $\mathbb{T}_{n}$ as the set of constraints in the optimization (14) for player $n$, and $\mathbb{T}=\left\{\mathbb{T}_{n}, \forall n\right\}$. Players' optimal strategies and the payoff values should therefore be $\mathbb{T}$-feasible solutions of the optimization problem.

\section{B. Equilibrium solution}

The Nash equilibrium can be defined as a joint strategy profile $\mathbf{L}^{*}=\left\{\mathbf{L}_{1}^{*}, \mathbf{L}_{2}^{*}, \ldots, \mathbf{L}_{N}^{*}\right\}, \mathbf{L}^{*} \in \mathbb{S}$, where $\mathfrak{u}_{n}\left(\mathbf{L}_{n}^{*}, \mathbf{L}_{-n}^{*}\right) \geq$ $\mathfrak{u}_{n}\left(\mathbf{L}_{n}, \mathbf{L}_{-n}^{*}\right), \forall \mathbf{L}_{n} \in \mathbb{S}_{n}, \forall n \in \mathbb{N}$, i.e., given the equilibrium strategy choices of other players $\mathbf{L}_{-n}^{*}$, player $n$ has no incentive to change his/her own strategy from $\mathbf{L}_{n}^{*}$ unilaterally [37]. Nash equilibrium is critical to the non-cooperative game theoretic modeling because, if it exists, it guarantees a stable solution where every player plays the best response to the strategic choices of all other players and the players have no incentive to deviate from this equilibrium.

As for the particular constrained scheduling optimization game, the Nash equilibrium will be the strategy profile which has the above property and also $\mathbb{T}$-feasible. Its existence will ensure that the scheduling process will be able to provide every consumer an optimal consumption scheduling. Different from the constrained games as discussed in [39] which will always guarantee a Nash equilibrium in pure strategies, the constrained solution is no longer in a continuous space due to the integer nature of the optimization of the payoff function. It is useful to adopt potential game approach to establish Nash equilibrium solution for the scheduling game.

\section{Potential game}

For a game with a set of players $\mathbb{N}$, feasible strategy space $\mathbb{S}$ and payoff functions $\mathfrak{u}_{n}\left(\mathbf{L}_{n}, \mathbf{L}_{-n}\right)$, a function $\mathfrak{U}: \mathbb{S} \rightarrow \mathbb{R}$ is called an exact potential function, if the following holds:

$$
\begin{gathered}
\mathfrak{u}_{n}\left(\mathbf{L}_{n}, \mathbf{L}_{-n}\right)-\mathfrak{u}_{n}\left(\mathbf{L}_{n}^{\prime}, \mathbf{L}_{-n}\right)=\mathfrak{U}\left(\mathbf{L}_{n}, \mathbf{L}_{-n}\right)-\mathfrak{U}\left(\mathbf{L}_{n}^{\prime}, \mathbf{L}_{-n}\right), \\
\forall n \in \mathbb{N}, \forall \mathbf{L}_{n}, \mathbf{L}_{n}^{\prime} \in \mathbb{S}_{n}, \forall \mathbf{L}_{-n} \in \mathbb{S}_{-n}, \mathbf{L}_{n} \neq \mathbf{L}_{n}^{\prime} .
\end{gathered}
$$

If a game admits an exact potential function which reflects the changes in the strategy chosen by any of the players, it falls into a specific class of strategic games called exact potential games [40]. It is observed that in the proposed scheduling game, although individual players are responsible for optimising only their own consumptions, the goal for all players is the minimization of the total cost. Any changes in the scheduling $\mathbf{L}_{n}$ will result in a change in the total cost, i.e.,

$$
\begin{gathered}
\mathfrak{u}_{n}\left(\mathbf{L}_{n}, \mathbf{L}_{-n}\right)-\mathfrak{u}_{n}\left(\mathbf{L}_{n}^{\prime}, \mathbf{L}_{-n}\right)=-\left(C(\mathbf{L})-C\left(\mathbf{L}^{\prime}\right)\right) \\
\forall n \in \mathbb{N}, \forall \mathbf{L}_{n}, \mathbf{L}_{n}^{\prime} \in \mathbb{S}_{n}, \forall \mathbf{L}_{-n} \in \mathbb{S}_{-n}, \mathbf{L}_{n} \neq \mathbf{L}_{n}^{\prime}
\end{gathered}
$$

Therefore $\mathfrak{U}=-C(\mathbf{L})$ is used as the exact potential function and the scheduling game is an exact potential game.

The theorem proposed in [40], which is very important for establishing Nash equilibrium for potential games, states that the potential game admits a pure strategy Nash equilibrium $\mathbf{L}^{*}$ if and only if $\mathbf{L}^{*}$ is a maximiser of the potential function. In other words, establishing Nash equilibrium of the scheduling game is equivalent to determining the solution of the constrained maximization of the potential function. The maximum is derived through an iterative best response convergence process. 


\section{Game procedure and iterative convergence}

A best response iterative process is a dynamic process that players update their actions by choosing the strategies that maximise their payoffs, given other players' current strategies remain fixed [41]. The best response $\mathfrak{B}_{n}^{m}\left(\mathbf{L}_{-n}^{m}\right)$ of player $n$ to the strategies $\mathbf{L}_{-n}^{m}$, where $m$ is the game iteration indicator starting from 0 , is given by

$$
\mathfrak{B}_{n}^{m}\left(\mathbf{L}_{-n}^{m}\right)=\underset{\mathbf{L}_{n} \in \mathbb{S}_{n}}{\arg \max } \mathfrak{u}_{n}\left(\mathbf{L}_{n}, \mathbf{L}_{-n}^{m}\right)
$$

Player $n$ will update its strategy to a new strategy $\mathbf{L}_{n}^{m+1} \in$ $\mathfrak{B}_{n}^{m}$ if and only if the new strategy gains an improvement to the payoff, i.e.,

$$
\mathfrak{u}_{n}\left(\mathbf{L}_{n}^{m+1}, \mathbf{L}_{-n}^{m}\right)>\mathfrak{u}_{n}\left(\mathbf{L}_{n}^{m}, \mathbf{L}_{-n}^{m}\right) .
$$

For the scheduling potential game, players will be able to carry out this process in a round robin manner. At each play, the player will have the opportunity to revise his/her scheduling with the aim of reducing the cost of energy consumption, which is equivalent to increasing the potential payoff. If the revised payoff is higher than the payoff obtained from the previous play, the player will play the revised new strategy, otherwise, the old strategy will be retained. Observing this move, the player at the next turn will optimise his/her strategy with the aim of further increasing the potential payoff. At every game iteration, the value of the potential function satisfies

$$
\mathfrak{U}\left(\mathbf{L}^{m+1}\right) \geq \mathfrak{U}\left(\mathbf{L}^{m}\right) .
$$

As the players keep optimising their strategies, the best response dynamics will result into a non-decreasing sequence of changes in the potential payoff $\left\{\mathfrak{U}\left(\mathbf{L}^{0}\right), \mathfrak{U}\left(\mathbf{L}^{1}\right), \mathfrak{U}\left(\mathbf{L}^{2}\right), \ldots\right\}$. This is called 'improvement path' which will finish at a point where no player will see any improvement in the payoff. At this point, the potential function $\mathfrak{U}$ will have converged to the maximum, which is the Nash equilibrium of the game. Since the cost is bounded above zero, i.e., it is non-negative, and its value will be changing non-increasingly within the game process, the convergence of the sequential game is guaranteed. In conclusion, the consumption scheduling game admits a $\mathbb{T}$-feasible Nash equilibrium in pure strategies.

\begin{tabular}{l}
\hline \hline Algorithm 1: Game procedure \\
\hline \hline Initialization: Each player generates its intended \\
consumption schedule according to its preferences as \\
initial strategy and broadcasts it to other players. \\
On detection of a new pricing signal, execute: \\
For $n=1: N$ \\
Player $n$ solves optimization in $(14)$ and obtains \\
the cost $C_{(n)}(\mathbf{L})$ and scheduling $\mathbf{L}_{n}$. \\
If the optimised scheduling $\mathbf{L}_{n}$ is different from the \\
previous scheduling strategy \\
$\quad$ Broadcast the new strategy $\mathbf{L}_{n}$ to other players. \\
else \\
Remain silent, i.e., no need for broadcasting $\mathbf{L}_{n}$. \\
End If \\
End For \\
Repeat For until no further improvement to all players. \\
\hline \hline
\end{tabular}

The scheduling game is expected to start every time a new pricing plan is issued. In order to play the best strategy $\mathbf{L}_{n}$ and to obtain maximal payoff, a player needs information which informs the player the current game status and the chosen strategies of other players $\mathbf{L}_{-n}$. A home energy management unit (EMU) is responsible for collecting and scheduling the consumption requirements. It serves as a data access point for scheduling information exchange during the game process. ICT infrastructure as in neighborhood area networks (NAN) and local area networks (LAN) can be used in smart grids to enable efficient and reliable communications among players. Candidate solutions include wireless 3G/LTE cellular and the emerging IEEE 802.22 which uses cognitive radio technologies in the white spaces of the television spectrum [1]. At the beginning of the game, every player should initialize a consumption schedule according to his/her own preference and announce it through the communication network. Acknowledging this information, the players will start to adjust their energy consumption plan using the best response process. The game theoretic algorithm for scheduling has been summarised in Algorithm 1.

\section{E. Efficiency, Complexity and Privacy}

The efficiency of the equilibrium solution of the game theoretic algorithm is often measured by Pareto optimality. Particularly for this game, a weaker version called constrained Pareto optimality is considered because all outcomes must be $\mathbb{T}$-feasible [42]. By definition, a strategy profile is Pareto optimal if there is no other profile that makes every player at least as well off while making at least one player strictly better off [43]. It can be claimed that the outcome of Algorithm 1 is automatically constrained Pareto optimal since it maximises the potential payoff which reflects the payoff for every player.

One of the important benefits of the proposed distributed approaches is that the computational complexity can be distributed among various home energy management units by decomposing the large scale centralised optimization using a decentralised game theoretic method. In terms of exchange of information, the distributed algorithms can also be efficient as compared to centralised algorithms. For example the distributed design approach in wireless networks enables coordinated beamforming without the need of explicit interbase-station information exchange as in [44], and the game theoretic approach as in [45]. For the demand management to be performed centrally, each household needs to inform the centralised scheduling processor the type of each appliance, its energy consumption pattern, consumer's preferred time of use, the use of electric vehicle and the availability of local energy, etc. This may require extensive amount of explicit data exchange between households and the centralised processor. For the proposed game theoretic approach, details on individual appliances are not required to be exchanged. As can be observed in Algorithm 1, the optimizations of detailed appliance consumption are done locally at every iteration, only the overall consumption profile $\mathbf{L}_{n}$ is required to be exchanged among players. This significantly reduces the 
amount of information exchange. In addition, the distributed approach has the benefit of enhanced privacy because the exact details of appliances and the information of individual household's locally generated energy are not exchanged. Only the aggregated use of energy is exchanged which has relatively lower private information. However, such information needs to be communicated among all players repeatedly during the iterative updating process. This may turn out to increase the communications overhead, especially for large number of consumers. Moreover, a centralised processor may possibly have more computational capacity to perform complex optimizations, hence centralised processer may be advantageous if there is any limitations on the computation and communication capability of the distributed EMUs. These tradeoffs need to be considered carefully in the choice between centralised and decentralised optimization approaches.

\section{F. Behaviour of players and their participation in the game}

The capacity of local energy supply is critical to the players in order to decide whether to participate in the scheduling game or not. Consider a particular consumer whose local capacity exceeds his daily consumption requirement. The consumer has the ability to self-supply his own energy demand and will achieve zero energy cost charged by the utility company even without any scheduling. In this case, as there is no impact in terms of pricing, this consumer has no incentive to participate in the scheduling game until there is a need to draw power from the main grid again. These players need not to play the game because scheduling does not provide any benefit to them. By observing the capacity of local energy supply, consumers should be able to decide whether to participate in the game immediately.

In non-cooperative games, there is a possibility that certain players may be untruthful during the game process by providing false information to the scheduling results. This will make the optimizations invalid and unable to reach the optimal result. Since the consumption optimization promises a lowest group cost and an optimal scheduling at the Nash equilibrium for every player in the game, there should be no incentive for players to cheat. However cheating could still occur when there are malicious players who always cheat intentionally to hurt others. Considering that the demand management activities will be carried out repeatedly on a daily basis, cheating players can be punished in future plays using various mechanisms as proposed for repeated games as in [37]. Developing workable mechanisms for detecting and preventing untruthful players is an open research topic.

\section{Simulations AND PERFORMANCE EVALUATION}

\section{A. System set up}

This section presents an illustrative simulation for the consumption scheduling optimization over one day using the proposed game theoretic approach. Consider a small residential area consisting of $N=10$ individual households. Each household has a set of appliances such as a 24 hour operational fridge $\&$ freezer (hourly consumption of $0.12 \mathrm{kWh}$ ) and electric heating (daily requirement of $4 \mathrm{kWh}$ ) with multiple

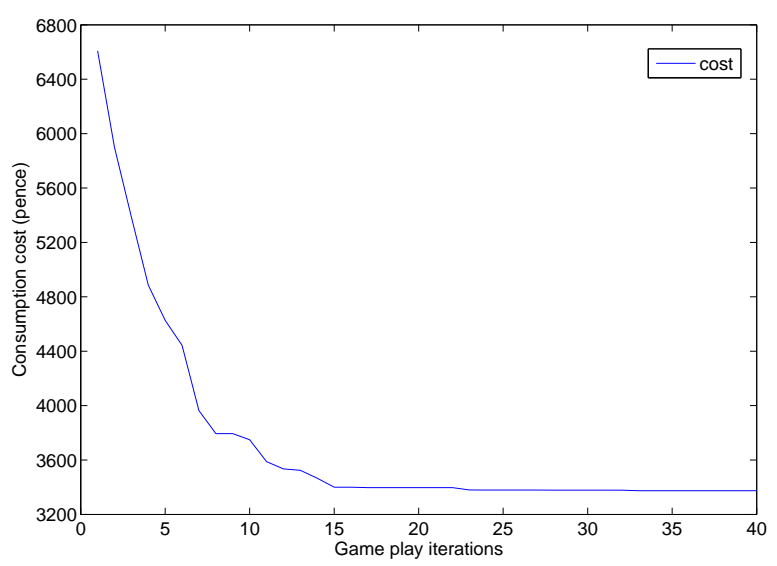

Fig. 2: Convergence of the scheduling game (played sequentially by each player), e.g., iteration 20 means each player played twice.

non-shiftable operations, washing machine with one shiftable operation program (daily requirement of $1.2 \mathrm{kWh}$ ) and a water tank boiler (daily consumption of $1.6 \mathrm{kWh}$ ) with multiple shiftable tasks over the day. The consumption requirements for these appliances have been obtained from [46] and [47]. Assume that the size of each household is different. The consumption requirements of the appliances and the total daily demands could vary. The daily demand of the 10 households is set at $256 \mathrm{kWh}$. User preferences for particular appliances also vary individually. In order to study the performance of the proposed consumption scheduling, the capacity of the local energy supply is assumed to be a small fraction chosen randomly between 15 percent and 25 percent of the daily requirement of each consumer so that every one will have incentive to participate in the game.

The pricing plan used in the simulation is based on a basic hourly ToU rate, hence the scheduling time resolution is one hour $(T=24)$. The unit rate $\omega_{t}$ is assumed to have the lowest value of one pence during the night period while the daytime rate is two pence and the peak rate of five pence appears in the morning between 9am and 10am and in the evening between $6 \mathrm{pm}$ and $8 \mathrm{pm}$. Assume there was zero standard admission charge $\phi_{t}$.

\section{B. Performance evaluation}

The convergence of the cost function against iteration of the game process is shown in Fig. 2. It is observed that the cost has dropped considerably fast during the first 10 iterations, i.e., when all the players finished their moves in the first round. The cost continued to reduce gradually and then became steady at 3374 pence after 20 plays. As the game went on for another two rounds, no player was able to reduce the cost further (improve the payoff). Therefore, after 30 iterations of individual scheduling, the game process is considered as finished and reached the Nash equilibrium point. Compared to rather high initial cost of 6586 pence, the proposed mechanism offered a significant reduction of nearly 50 percent of the total cost. As seen, the convergence of the scheduling game is reasonably fast. As for this simulation scenario, it is claimed that only three rounds of play per player is adequate for the game to reach stable NE point. 
In order to evaluate the benefits for individual consumers, the cost of energy consumption for each consumer has been provided in Table I. Compared to the cost without scheduling, every consumer gains a cost reduction of around 50 percent by participating in the optimization game, which is fairly identical to the overall cost reduction. In other words, the overall benefits have been fairly distributed to all players who participated in the game.

\begin{tabular}{|c|c|c|c|}
\hline & $\begin{array}{c}\text { Without } \\
\text { scheduling }\end{array}$ & $\begin{array}{l}\text { Game } \\
\text { result }\end{array}$ & $\begin{array}{c}\text { Isolated } \\
\text { scheduling }\end{array}$ \\
\hline Consumer 1 & 433.80 & 227.83 & 258.03 \\
\hline Consumer 2 & 595.57 & 258.01 & 294.83 \\
\hline Consumer 3 & 609.21 & 307.26 & 323.70 \\
\hline Consumer 4 & 689.55 & 342.26 & 375.44 \\
\hline Consumer 5 & 515.02 & 283.71 & 305.93 \\
\hline Consumer 6 & 694.08 & 412.96 & 444.00 \\
\hline Consumer 7 & 839.80 & 389.16 & 445.40 \\
\hline Consumer 8 & 780.08 & 400.42 & 461.41 \\
\hline Consumer 9 & 879.62 & 499.94 & 502.80 \\
\hline Consumer 10 & 548.25 & 253.05 & 292.32 \\
\hline Total & 6584.96 & 3374.60 & 3703.85 \\
\hline
\end{tabular}

Table I compares the cost with the result of an isolated scheduling scheme where players are assumed to have the same appliances and user preferences as in the game above, however they schedule their consumption independently according to the price plan, without knowing others' scheduling information. As seen, an average cost reduction of 35 pence has been achieved for each game participant. Therefore, the proposed game theoretic framework has the ability to reduce the cost by encouraging consumers to participate. The difference in performance is expected to become considerably high when a large number of subscribers are involved.

Fig. 3 depicts the scheduled optimal consumption for all the participating households after the game has converged. It is observed that the consumptions remain reasonably low during the day time and the majority of the demand has been shifted to the night time corresponding to lower price. Most of the local energies were drawn to further reduce the potential high demand, in particular at peak times in the morning and in the evening. The consumption optimization performed effectively in both integrating local energy resource optimally and also scheduling possible consumption away from the peak ToU periods. It should be noted that even though the terminology of balanced scheduling is used, it is not expected to achieve equal consumption distribution throughout the day. This is because the aim is not to distribute the demand equally, but to fit it to the expectation of the utility operator according to the price profile, i.e., to move most of energy consumptions to off peak period as seen in Fig. 3. The reason for this is that the demand might be high, for example due to industrial use of electricity in the day time, and the aim of utility operator is to balance the overall demand consisting of both the industrial and residential demands, by issuing appropriate pricing plans. The game theoretic method proposed helps to ensure the consumers do not operate all the appliances at the same time, but distribute over the time as much as possible, as seen in the results.

Table II lists the usage of local energy for each player.

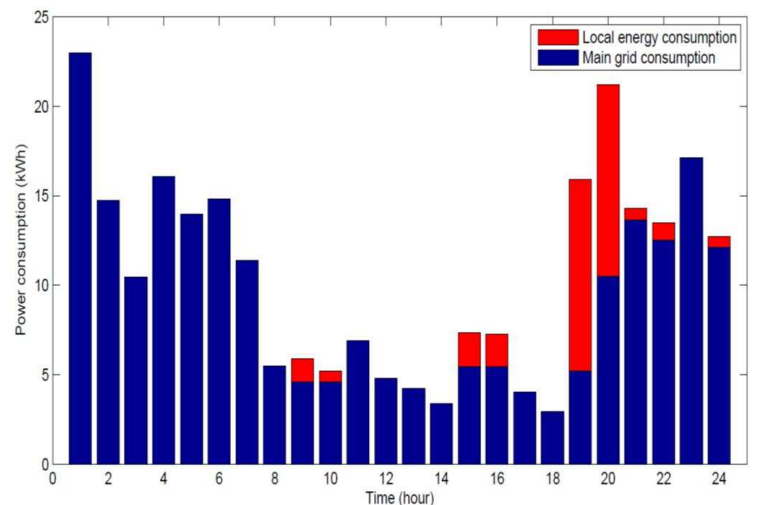

Fig. 3: Scheduled accumulated energy consumption over the day.

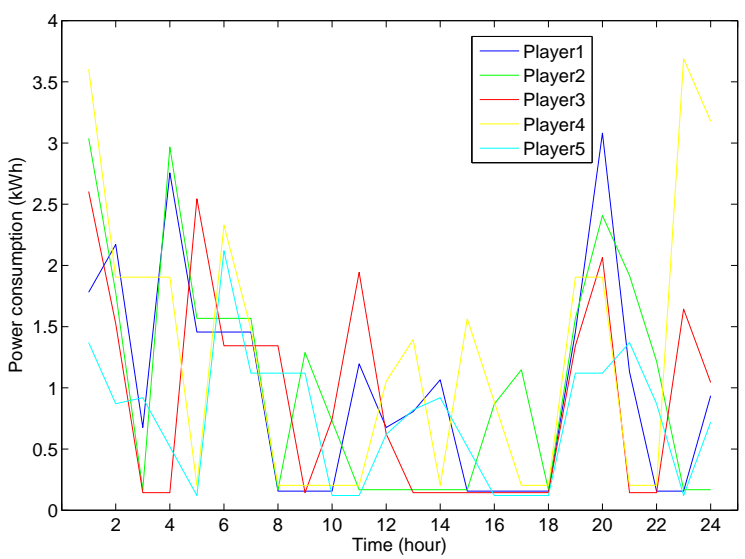

Fig. 4: Comparison of scheduled consumption for five players.

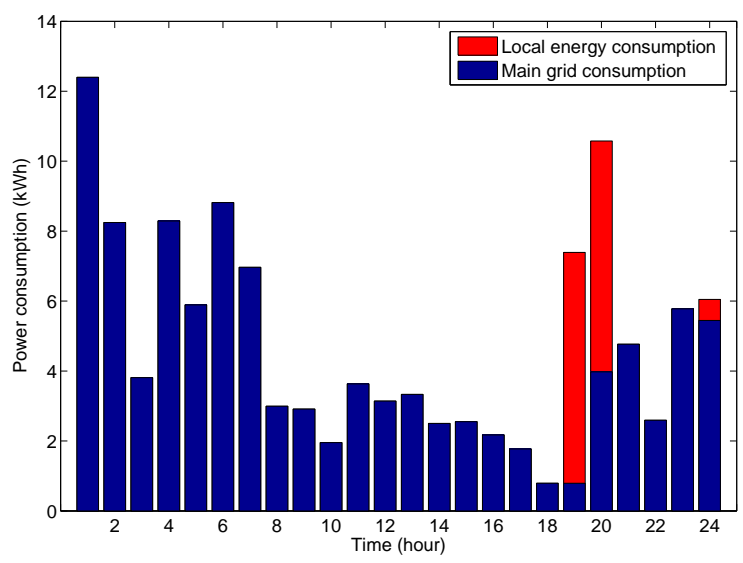

Fig. 5: Scheduled consumption from local and main supply for five players.

The majority of local capacity has been scheduled to be used. However, it appears that there is still a small portion of energy that was not utilised. This is because this energy was not being able to be served to any of the appliance on the current date of scheduling. This unused energy can be stored for future use.

\begin{tabular}{|c|c|c|c|}
\hline & Usage & & Usage \\
\hline Consumer 1 & $87 \%$ & Consumer 6 & $96 \%$ \\
\hline Consumer 2 & $96 \%$ & Consumer 7 & $85 \%$ \\
\hline Consumer 3 & $87 \%$ & Consumer 8 & $90 \%$ \\
\hline Consumer 4 & $93 \%$ & Consumer 9 & $86 \%$ \\
\hline Consumer 5 & $90 \%$ & Consumer 10 & $95 \%$ \\
\hline
\end{tabular}

Fig. 4 compares the consumption schedules of five individual consumers. As seen, the peak energy consumption of 
consumers occurs at different time slots. This implies the shiftable operations have been well distributed with minimal overlapping with each other. However, considerable overlapping still appeares, e.g., in the hours between $7 \mathrm{pm}$ and $8 \mathrm{pm}$. This is caused due to the non-shiftable requirements during the peak hours in the evening. As depicted in Fig. 5, the local energy supplies were mainly scheduled at these periods in order to reduce the dependency on the main grid to prevent high accumulated consumption and cost. The chargeable consumption has decreased by approximately 70 percent between the hours of $7 \mathrm{pm}$ and $8 \mathrm{pm}$, i.e., the effect of overlapping was signigicantly reduced. In summary, through the scheduling game, consumers are able to optimally manage their daily consumption and integrate local generated/stored energy.

\section{CONCLUSIONS}

This paper presented a non-cooperative game theoretic consumption scheduling framework based on MIP optimization technique to schedule the energy consumption at household level. The scheme has the ability to reduce energy cost and the accumulated consumption. Consumption requirements of the appliances were classified into different groups and formulated into the optimization. In addition, locally generated renewable energy has been integrated into the consumption scheduling optimization to further reduce the demand on conventional energy. The proposed game admits a Nash equilibrium where the scheduling optimization process finds a stable solution at which every consumer benefits from minimised cost of energy consumption. The simulations demonstrated the convergence of the algorithm and the benefits to the consumers and the grid operators.

\section{REFERENCES}

[1] Z. Zhu, S. Lambotharan, W. H. Chin, and Z. Fan, "Overview of demand management in smart grid and enabling wireless communication technologies," IEEE Wireless Communications, vol. 19, pp. 48-56, June 2012.

[2] European Commission, "European Smart Grids Technology Platform Vision and Strategy for Europes Electricity Networks of the Future," Directorate-General for Research - Sustainable Energy Systems, 2006, Available http://www.smartgrids.eu.

[3] J. Aghaei and M.-I. Alizadeh, "Demand response in smart electricity grids equipped with renewable energy sources: A review," Renewable and Sustainable Energy Reviews, vol. 18, p. 6472, 2013.

[4] Z. Fan, P. Kulkarni, S. Gormus, C. Efthymiou, G. Kalogridis, M. Sooriyabandara, Z. Zhu, S. Lambotharan, and W. Chin, "Smart grid communications: Overview of research challenges, solutions, and standardization activities," Communications Surveys Tutorials, IEEE, vol. PP, no. 99, pp. 1-18, 2012.

[5] P. Siano, "Demand responseandsmartgridsa survey," Renewable and Sustainable Energy Reviews, vol. 30, p. 461478, 2014.

[6] C. Gellings and W. Smith, "Integrating demand-side management into utility planning," Proceedings of the IEEE, vol. 77, no. 6, pp. 908-918, 1989.

[7] H. Zareipour, A. Janjani, H. Leung, A. Motamedi, and A. Schellenberg, "Electricity price thresholding and classification," in 2011 IEEE Power and Energy Society General Meeting, pp. 1-7, July 2011.

[8] H. Zhong, L. Xie, and Q. Xia, "Coupon incentive-based demand response: Theory and case study," IEEE Transactions on Power Systems, vol. 28, pp. 1266-1276, May 2013.

[9] J. Aghaei and M.-I. Alizadeh, "Critical peak pricing with load control demand response program in unit commitment problem," IET Generation, Transmission Distribution, vol. 7, pp. 681-690, July 2013.

[10] H. Chao, "Competitive electricity markets with consumer subscription service in a smart grid," Journal of Regulatory Economics, vol. 41, no. 1, pp. 155-180, 2012.
[11] R. Yu, W. Yang, and S. Rahardja, "A statistical demand-price model with its application in optimal real-time price," IEEE Transactions on Smart Grid, vol. 3, pp. 1734-1742, Dec 2012.

[12] M. Albadi and F. El-Saadany, "Demand Response in Electricity Markets: An Overview," in IEEE Power Engineering Society General Meeting, 2007.

[13] R. Weron, Modeling and Forecasting Electricity Loads and Prices: A Statistical Approach. New York: Wiley, 2006.

[14] T. Lui, W. Stirling, and H. Marcy, "Get smart," IEEE Power and Energy Magazine, vol. 8, pp. 66-78, May-June 2010.

[15] G. Koutitas and L. Tassiulas, "A delay based optimization scheme for peak load reduction in the smart grid," in Future Energy Systems: Where Energy, Computing and Communication Meet (e-Energy), 2012 Third International Conference on, pp. $1-4$, may 2012.

[16] T. Logenthiran, D. Srinivasan, and T. Z. Shun, "Demand side management in smart grid using heuristic optimization," IEEE Transactions on Smart Grid, vol. 3, pp. $1244-1252$, sept. 2012.

[17] Y. Ozturk, D. Senthilkumar, S. Kumar, and G. Lee, "An intelligent home energy management system to improve demand response," IEEE Transactions on Smart Grid, vol. 4, pp. 694-701, June 2013.

[18] A. Zakariazadeh, S. Jadid, and P. Siano, "Smart microgrid energy and reserve scheduling with demand response using stochastic optimization," Electrical Power and Energy Systems, vol. 63, pp. 523-533, 2014.

[19] J. Aghaei and M.-I. Alizadeh, "Multi-objective self-scheduling of CHP (combined heat and power)-based microgrids considering demand response programs and ESSs(energy storage systems)," Energy, vol. 55, pp. 1044-1054, 2013.

[20] A. Zakariazadeh, S. Jadid, and P. Siano, "Economic-environmental energy and reserve scheduling of smart distribution systems: A multiobjective mathematical programming approach," Energy Conversion and Management, vol. 78, pp. 151-164, 2014.

[21] S. Lasaulce, M. Debbah, and E. Altman, "Methodologies for analyzing equilibria in wireless games," IEEE Signal Processing Magazine, vol. 26, pp. 41-52, Sept. 2009.

[22] G. Scutari, D. Palomar, F. Facchinei, and J. shi Pang, "Convex optimization, game theory, and variational inequality theory," IEEE Signal Processing Magazine, vol. 27, pp. 35-49, May 2010.

[23] W. Saad, Z. Han, H. Poor, and T. Basar, "Game-theoretic methods for the smart grid: An overview of microgrid systems, demand-side management, and smart grid communications," IEEE Signal Processing Magazine, vol. 29, no. 5, pp. 86-105, Sept.2012.

[24] A. Mohsenian-Rad, V. Wong, J. Jatskevich, R. Schober, and A. LeonGarcia, "Autonomous demand-side management based on gametheoretic energy consumption scheduling for the future smart grid," IEEE Transactions on Smart Grid, vol. 1, no. 3, pp. 320-331, Dec. 2010.

[25] Q. Zhu, Z. Han, and T. Basar, "A differential game approach to distributed demand side management in smart grid," in IEEE International Conference on Communications, pp. 3345 -3350, june 2012.

[26] S. Maharjan, Q. Zhu, Y. Zhang, S. Gjessing, and T. Basar, "Dependable demand response management in the smart grid: A stackelberg game approach," IEEE Transactions on Smart Grid, vol. 4, p. 120132, 2013.

[27] Z. Chen, L. Wu, and Y. Fu, "Real-time price-based demand response management for residential appliances via stochastic optimization and robust optimization," IEEE Transactions on Smart Grid, vol. 3, pp. 1822-1831, Dec 2012.

[28] N. Li, L. Chen, and S. Low, "Optimal demand response based on utility maximization in power networks," in IEEE Power and Energy Society General Meeting, pp. 1-8, July 2011.

[29] I. Koutsopoulos and L. Tassiulas, "Control and optimization meet the smart power grid-scheduling of power demands for optimal energy management," in Proceedings of 2nd International Conference on EnergyEfficient Computing and Networking (E-Energy), 2011.

[30] Z. Wang and F. Li, "Critical peak pricing tariff design for mass consumers in great britain," in 2011 IEEE Power and Energy Society General Meeting, pp. 1-6, July 2011.

[31] C. Li, S. Tang, Y. Cao, Y. Xu, Y. Li, J. Li, and R. Zhang, "A new stepwise power tariff model and its application for residential consumers in regulated electricity markets," IEEE Transactions on Power Systems, vol. PP, no. 99, p. 1, 2012.

[32] F. Li, "Continuous locational marginal pricing (CLMP)," IEEE Transactions on Power Systems, vol. 22, pp. 1638 -1646, Nov. 2007.

[33] M. Greer, Electricity Cost Modeling Calculations. Elsevier Science, 2010.

[34] B. Li, S. Gangadhar, S. Cheng, and P. Verma, "Maximize user rewards in distributed generation environments using reinforcement learning," in IEEE Energytech, pp. 1-6, may 2011. 
[35] Z. Zhu, J. Tang, S. Lambotharan, W. H. Chin, and Z. Fan, "An integer linear programming based optimization for home demand-side management in smart grid," in IEEE PES ISGT, Washington DC, USA, Jan. 2012

[36] G. L. Nemhauser and L. A. Wolsey, Integer and combinatorial optimization. John Wiley and Sons, New York, 1998.

[37] M. J. Osborne, An introduction to game theory. Oxford University Press, 2004.

[38] N. Nisan, T. Roughgarden, E. Tardos, and V. V. Vazirani, Algorithmic Game Theory. UK: Cambridge University Press, 2007.

[39] J. Rosen, "Existence and uniqueness of equilibrium points for concave n-person games," Econometrica, vol. 33, no. 3, pp. 520-534, 1965.

[40] D. Monderer and L. S. Shapley, "Potential games," Games and Economic Behavior, vol. 14, pp. 124-143, 1996.

[41] M. Voorneveld, "Economic letters," Best-response potential games, vol. 66, pp. 289-295, 2000.

[42] D. L. Brito, J. H. Hamilton, S. M. Slutsky, and J. E. Stiglitz, "Pareto efficient tax structures," Oxford Economic Papers, vol. 42, pp. 61-77, 1990.

[43] S. Boyd and L. Vandenberghe, Convex Optimization. Cambridge University Press, Cambridge, UK, 2004.

[44] H. Dahrouj and W. Yu, "Coordinated beamforming for the multicell multi-antenna wireless system," Wireless Communications, IEEE Transactions on, vol. 9, no. 5, pp. 1748-1759, 2010.

[45] D. H. N. Nguyen and T. Le-Ngoc, "Multiuser downlink beamforming in multicell wireless systems: A game theoretical approach," IEEE Transactions on Signal Processing, vol. 59, no. 7, pp. 3326-3338, 2011.

[46] EERE. US Department of Energy. http://www.eere.energy.gov/.

[47] UK Department of Energy \& Climate Change. http://www.decc.gov.uk/. 